\title{
The XXL survey: First results and future
}
M. Pierre ${ }^{*}$ | C. Adami ${ }^{2}$ | M. Birkinshaw ${ }^{3}$
L. Chiappetti ${ }^{4}$
S. Ettori ${ }^{5}$
A. Evrard 6
L. Faccioli ${ }^{1}$ |
F. Gastaldello 4
P. Giles ${ }^{3}$ |
C. Horellou ${ }^{7}$
A. Iovino $^{8}$ | E. Koulouridis ${ }^{1}$ |
C. Lidman'
A. Le Brun ${ }^{1}$
B. Maughan 3
S. Maurogordato ${ }^{10}$
I. McCarthy ${ }^{11}$ ।
S. Miyazaki ${ }^{12}$
F. Pacaud ${ }^{13}$
S. Paltani ${ }^{14}$
M. Plionis ${ }^{15}$ ।
T. Reiprich $^{13}$ | T. Sadibekova ${ }^{1,16}$ |
V. Smolcic ${ }^{17}$
S. Snowden ${ }^{18}$ ।
J. Surdej ${ }^{19}$
M. Tsirou ${ }^{1}$
C. Vignali ${ }^{56}$
J. Willis ${ }^{20}$
S. Alis ${ }^{21}$
B. Altieri ${ }^{22}$
N. Baran ${ }^{17}$ |
C. Benoist ${ }^{10}$
A. Bongiorno ${ }^{23}$
M. Bremer ${ }^{3}$

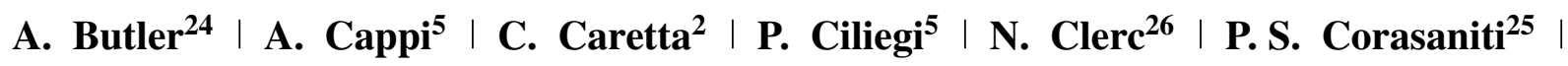

J. Coupon ${ }^{14}$ ।

J. Delhaize ${ }^{17}$

I. Delvecchio ${ }^{17}$

J. Democles ${ }^{30}$

Sh. Desai ${ }^{27}$

J. Devriendt ${ }^{28}$ | Y. Dubois ${ }^{28}$ |

D. Eckert $^{14}$ ।

A. Elyiv ${ }^{19}$

A. Farahi $^{29}$

C. Ferraril ${ }^{10}$

S. Fotopoulou ${ }^{14}$

W. Forman ${ }^{30}$

I. Georgantopoulos ${ }^{31}$

V. Guglielmo ${ }^{32}$

M. Huynh 24

N. Jerlin ${ }^{17}$

Ch. Jones ${ }^{30}$

S. Lavoie $^{20}$

J.-P. Le Fevre F3 $^{33}$

M. Lieu ${ }^{22}$

M. Kilbinger ${ }^{1}$ ।

F. MaruIli56 ।

A. Mantz ${ }^{34}$ ।

S. $\mathrm{McGee}^{38}$

J.-B. Melin ${ }^{36}$

O. Melnyk ${ }^{17}$

L. Moscardini56

M. Novak ${ }^{17}$ | E. Piconcelli ${ }^{23}$

B. Poggianti ${ }^{32}$

D. Pomarede P3 $^{33}$

E. Pompei ${ }^{37}$

T. Ponman ${ }^{38}$

M. E. Ramos Ceja ${ }^{13}$ | P. $\operatorname{Rana}^{39} \mid$ D. Rapetti $^{40} \mid$ S. Raychaudhury ${ }^{38} \mid$ M. Ricci ${ }^{10}$ |

H. Rottgering ${ }^{35} \mid$ M. Sahlen ${ }^{29}$ | J.-L. Sauvageot ${ }^{1}$ | C. Schimd ${ }^{2} \mid$ M. Sereno S G.P. Smith $^{38}$ |

K. Umetsu ${ }^{41}$ | P. Valageas $^{42}$ | A. Valotti ${ }^{1}$ I. Valtchanov ${ }^{22}$ | A. Veropalumbo ${ }^{5}$ |

B. Ascaso $^{43}$ ।

D. Barnes ${ }^{44}$ ।

M. De Petris ${ }^{45}$

F. Durret ${ }^{46}$

M. Donahue ${ }^{6}$

| M. Ithana ${ }^{47}$ |

M. Jarvis ${ }^{28}$ |

M. Johnston-Hollitt ${ }^{48}$ |

E. Kalfountzou ${ }^{22}$

S. $\mathrm{Kay}^{44}$ ।

N. Okabe ${ }^{50}$ ।

A. Muzzin ${ }^{51}$

A. Rettura ${ }^{52}$

F. $\operatorname{Ricci}^{49}$

J. Ridl ${ }^{26}$

G. Risaliti ${ }^{53}$

M. Takizawa ${ }^{47}$

P. Thomas ${ }^{54}$

N. Truong ${ }^{55}$

${ }^{1}$ Service d'Astrophysique du CEA, Saclay, France

${ }^{2}$ Laboratoire d'Astrophysique, Marseille, France

${ }^{3}$ University of Bristol, Bristol, UK

${ }^{4}$ INAF-IASF, Milano, Italy

${ }^{5}$ INAF-OABO, Bologna, Italy

${ }^{6}$ University of Michigan, Ann Arbor, Michigan

${ }^{7}$ Chalmers University of Technology, Onsala,

Sweden

${ }^{8}$ INAF-OAB, Brera, Italy

${ }^{9}$ Australian Astronomical Observatory, Epping,

Australia

${ }^{10}$ Observatoire de la Cote d'Azur, Nice, France

${ }^{11}$ University of Liverpool, Liverpool, UK

${ }^{12}$ NAOJ, Tokyo, Japan

${ }^{13}$ Argelander-Institut fur Astronomie, Bonn,

Germany
The XXL survey currently covers two $25 \mathrm{deg}^{2}$ patches with XMM observations of $\sim 10 \mathrm{ks}$. We summarize the scientific results associated with the first release of the XXL dataset, which occurred in mid-2016. We review several arguments for increasing the survey depth to $40 \mathrm{ks}$ during the next decade of XMM operations. X-ray $(z<2)$ cluster, $(z<4)$ active galactic nuclei (AGN), and cosmic background survey science will then benefit from an extraordinary data reservoir. This, combined with deep multi- $\lambda$ observations, will lead to solid standalone cosmological constraints and provide a wealth of information on the formation and evolution of AGN, clusters, and the X-ray background. In particular, it will offer a unique opportunity to pinpoint the $z>1$ cluster density. It will eventually constitute a reference study and an ideal calibration field for the upcoming eROSITA and Euclid missions. 
${ }^{14}$ ISDC, Geneva Observatory, Switzerland

${ }^{15}$ Aristotle University, Thessaloniki, Greece

${ }^{16}$ Ulugh Beg Astronomical Institute, Tashkent,

Uzbekistan

${ }^{17}$ University of Zagreb, Croatia

${ }^{18}$ NASA, GSFC, Greenbelt, Maryland

${ }^{19}$ University of Liège, Belgium

${ }^{20}$ University of Victoria, Canada

${ }^{21}$ Istanbul University, Istanbul, Turkey

${ }^{22}$ European Space Astronomy Center, Madrid, Spain

${ }^{23}$ INAF-OAR, Roma, Italy

${ }^{24}$ University of Western Australia, Australia

${ }^{25}$ Observatoire de Paris, France

${ }^{26}$ MPI for Extraterrestrial Physics, Garching, Germany

${ }^{27}$ IIT Hyderabad, India

${ }^{28}$ University of Oxford, UK

${ }^{29}$ Uppsala University, Sweden

${ }^{30}$ HSCenter for Astrophysics, Cambridge,

Massachusetts

${ }^{31}$ Observatory of Athens, Greece

${ }^{32}$ INAF-OAP, Padova, Italy

${ }^{33}$ Service d'Informatique du CEA, Saclay, France

${ }^{34}$ University of Chicago, Chicago, Illinois

${ }^{35}$ Leiden University, Leiden, The Netherlands

${ }^{36}$ Service de Physique des Particules du CEA,

Saclay, France

${ }^{37}$ European Southern Observatory, Garching, Germany

${ }^{38}$ University of Birmingham, Birmingham, UK

${ }^{39}$ University of Lund, Lund, Sweden

${ }^{40}$ University of Colorado, Boulder, Colorado

${ }^{41}$ ASIAA, Taipei, Taiwan

${ }^{42}$ Institut de Physique Theorique du CEA, Saclay,

France

${ }^{43}$ University Paris-Diderot, Paris, France

${ }^{44}$ Jodrell Bank, Manchester, UK

${ }^{45}$ University La Sapienza, Rome, Italy

${ }^{46}$ Institut d'Astrophysique de Paris, Paris, France

${ }^{47}$ Yamagata University, Japan

${ }^{48}$ Victoria University, Wellington, New Zealand

${ }^{49}$ University Roma Tre, Rome, Italy

${ }^{50}$ Hiroshima University, Hiroshima, Japan

${ }^{51}$ York University, Toronto, Canada

${ }^{52}$ IPAC, Pasadena, California

${ }^{53}$ Arcetri Observatory, Florence, Italy

${ }^{54}$ University of Sussex, Brighton, UK

${ }^{55}$ University of Tor Vegata, Roma, Italy

${ }^{56}$ University of Bologna, Bologna, Italy

*Correspondence

M. Pierre, Service d'Astrophysique du CEA Saclay F-91190 Gif sur Yvette.

Email: mpierre@cea.fr

\section{KEYWORDS}

$\mathrm{X}$-ray: general, cosmological parameters, galaxies: clusters: general, galaxies: active

\section{1 | INTRODUCTION}

Almost 17 years after the launch of XMM-Newton, it is time to review its scientific achievements. A thorough census of the still-open or newly raised questions will help us to optimize the use of the observatory for its last decade. In this paper, we focus on medium-deep extragalactic surveys. More specifically, we scrutinize the contribution of X-ray large-scale structure studies to the global multi- $\lambda$ and multiprobe effort toward precision cosmology. Although XMM was not initiallygaski designed as a survey instrument, its large field of view, good point spread function (PSF), and unrivalled collecting area provide a unique opportunity to scan the structure of the energetic universe. The mosaic observing mode implemented in 2008 further enhanced these capabilities.

Starting from the Guaranteed Time pooled by the Liège, Milano, and Saclay groups at the very beginning of the XMM mission, we undertook a uniform mapping of the 
extragalactic sky. Subsequent Guest Observer observations of $\sim 10 \mathrm{ks}$ allowed us to achieve a coverage of some $11 \mathrm{deg}^{2}$ by 2009 (Chiappetti et al. 2013; Clerc et al. 2014; Elyiv et al. 2012). This XMM-LSS pilot survey was an essential step in understanding the $\mathrm{X}$-ray cluster selection function (down to a depth never explored to far) and in testing its impact on the scaling relations and subsequent cosmological analysis. In 2010, we were allocated an XMM Very Large Program to extend the coverage to two areas of $25 \mathrm{deg}^{2}$ each at the same sensitivity: the XXL survey. ${ }^{1}$

The main driver of the XXL survey is cosmology, based on both active galactic nuclei (AGN) and cluster counts along with three-dimension topological and environmental studies: hence the need for a large connected area, rather than serendipitous archival detections. Other fundamental motivations for promoting a large-scale, uniform X-ray coverage include the simplification of the selection function and the availability of a set of associated homogeneous surveys covering the entire electromagnetic spectrum on the same area (from ultraviolet [UV] to radio). This enables coherent source identification along with uniform spectral energy distribution (SED) and redshift measurements, which constitute the two fundamental steps toward the census of the cluster and AGN populations and their characterization.

In this paper, we first recall the main issues pertaining to cluster cosmology, and then summarize the outcome of the recent series of XXL articles. In the last sections, we propose a route for extending the current existing dataset and provide a truly outstanding scientific legacy.

\section{2 | CLUSTER COSMOLOGY AND THE MOTIVATIONS OF THE XXL PROJECT}

As the most massive self-gravitating entities of the universe, clusters of galaxies are theoretically key objects to constrain cosmological models: they are both sensitive to the geometry of the space-time and to structure growth. Originating from physical processes different from those of the cosmic microwave background (CMB), supernovae, and baryonic acoustic oscillations, they should provide independent and complementary constraints. However, a number of practical difficulties, most of them having been overlooked before the advent of XMM and Chandra, render such a study especially challenging. These include the following: (a) It is now well established that the $\mathrm{X}$-ray selection function of these extended objects cannot be modeled by a simple flux limit but should be estimated in the flux-size parameter plane. (b) Scaling relations, which enable the use of mass proxies (e.g., Lx, Tx Mgas, or the optical richness), are very much dependent on the samples on which they are based; disentangling the selection effects requires the knowledge of the intrinsic scatter of

${ }^{1}$ http://irfu.cea.fr/xxl

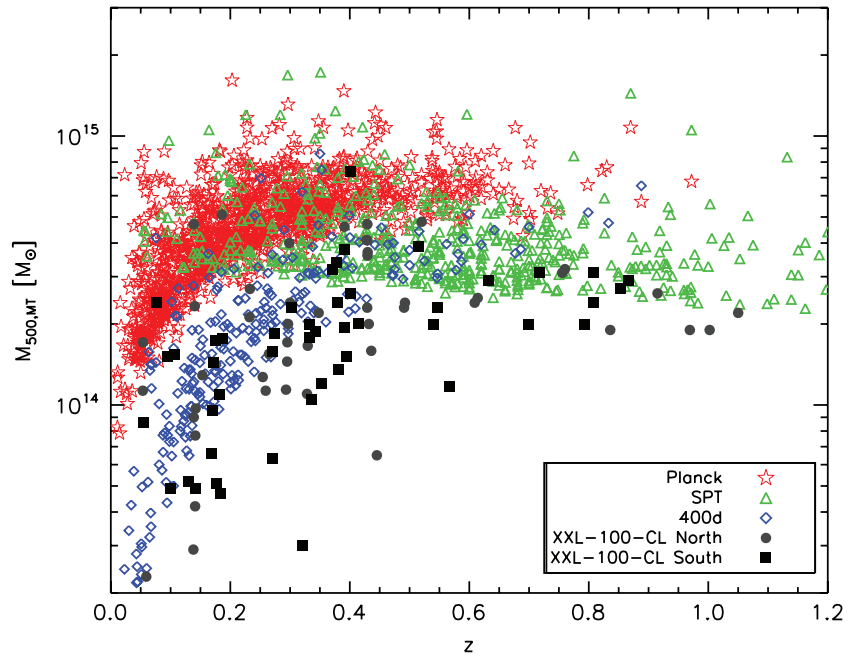

FIGURE 1 Mass range covered by the XXL brightest 100 clusters, compared to other surveys. Credit: Pacaud et al. (2016), reproduced with permission (C) ESO

these relations. However, very few scatter measurements exist and, most of the time, one relies on assumptions from numerical simulations. (c) The fact that cluster masses are not a direct observable continues to feed a lively controversy, motivating innovative observational studies. To this should be added that hydrodynamic simulations indicate a bias up to $20-30 \%$ between true and hydrostatic masses. (d) The whole picture must be consistently worked out in an evolving environment, while the evolution of the cluster baryonic physics is still very much debated. Rigorously, cosmology, cluster evolution, and selection effects should be addressed in a self-consistent approach (for a review on these topics, see, e.g., Allen et al. 2011)

In this context, the XXL survey aims at an independent and self-consistent cosmological analysis. As much as possible, scaling relations are derived from the cluster sample itself in conjunction with measurements in other wavebands like the integrated K-band luminosity or deep weak lensing information. The interplay between cluster and AGN physics, as well as its impact on cluster detection and scaling relations, is studied with great care via several sets of numerical simulations. Given its $50 \mathrm{deg}^{2}$ coverage, XXL tackles the very important, and still largely unexplored, $M_{500} \sim 5 \times 10^{13}-2 \times 10^{15} M_{\odot}$ regime for $z \sim 0.5$ clusters and thus provides information complementary to the Planck, SPT, and Weighing the Giants samples (Figure 1).

\section{3 | FIRST RESULTS FROM THE XXL SUR VEY}

The XXL survey gathers some 100 scientists worldwide and is accompanied by a comprehensive multi- $\lambda$ and spectroscopic program. The two surveyed $25 \mathrm{deg}^{2}$ areas $\left(\mathrm{XXL}-\mathrm{N}: \mathrm{RA}=2 \mathrm{~h} 30 \mathrm{Dec}=-4 \mathrm{~d} 30^{\prime} ; \mathrm{XXL}-\mathrm{S}: \mathrm{RA}=23 \mathrm{~h} 30\right.$ Dec $\left.=-55{\mathrm{~d} 00^{\prime}}^{\prime}\right)$ are covered by more than 500 independent 
TABLE 1 The XXL databases. As of the 2016 release, the X-ray catalogs are limited to the brightest 100 clusters and 1,000 active galactic nuclei (AGN). Incrementally deeper releases will follow. In addition, the database in Milano provides the X-ray raw and wavelet MOS+pn mosaic images as well as all exposure maps for both XXL fields up to AO11

\begin{tabular}{l|l}
\hline Clusters & http://xmm-lss.in2p3.fr:8080/xxldb/index.html \\
& X-ray and optical images \\
& Details of the redshift calculations \\
& X-ray: flux, luminosity, temperature \\
& Mass estimate \\
& http://cosmosdb.iasf-milano.inaf.it/XXL/ \\
\hline AGN & Fluxes, X-ray spectral fits, counterparts \\
& VLA 3GHz and ATCA $2.1 \mathrm{GHz}$ radio catalogues \\
& AAOmega redshifts \\
\hline
\end{tabular}

XMM observations totaling some 6.9 Ms, which makes XXL the largest XMM program to date. It was designed so as to provide a sample of some 500 clusters of galaxies out to a redshift of unity, suitable for cosmological study. The point-source sensitivity is $\sim 5 \times 10^{-15} \mathrm{erg} \mathrm{s}^{-1} \mathrm{~cm}^{-2}$ in the $0.5-2 \mathrm{keV}$ band. The survey characteristics along with its extensive imaging+spectroscopic associated follow-up and simulation programs are presented in Pierre et al. (2016). In June 2016, the first series of XXL results was published in a special issue of Astronomy and Astrophysics. ${ }^{2}$ They are based on the brightest 100 clusters and 1,000 AGN samples. Both X-ray catalogs, along with two associated VLA and ATCA radio source lists, are available at the CDS. They can also be retrieved in a more extensive form, along with the XMM images and exposure maps, via the XXL databases (Table 1). The XXL team pays special attention to the delivery of well-validated catalogs and, beside the science publications, considers this legacy aspect a priority commitment.

\section{1 | Summary of the first results}

The 2016 results pertain to about one fifth and one twentith of the complete cluster and AGN samples, respectively. They already provide interesting clues, which can be summarized as follows:

\subsection{1 | Clusters}

(a) We performed an internally consistent derivation of the M-T and L-T relations (Giles et al. 2016; Lieu et al. 2016); (b) The luminosity function does not show evolution out to a redshift of unity (Pacaud et al. 2016) while the L-T relation is compatible with self-similar evolution (Giles et al. 2016); (c) The modeling of the cluster number counts shows a deficit with respect to predictions assuming the Planck CMB cosmology; (d) The low gas content of these clusters favors strong AGN feedback activity (Eckert et al. 2016); (e) We discovered five superclusters (Pacaud et al. 2016; Pompei et al. 2016); (f) We have detected via the Sunyaev-Zel'dovich effect (S-Z) one of the XXL distant cluster candidates, which turned out to be the highest redshift cluster $(z \sim 1.9)$ ever detected to date in S-Z (Mantz et al. 2014).

\subsection{2 | Active galactic nuclei}

(a) We improved upon the photometric redshift determination for AGN by applying a random forest classification trained to identify the optimal photometric redshift category for each object (passive, star-forming, starburst, AGN, QSO). (b) The X-ray spectral properties are consistent with those of the bright sources from the literature. (c) The $2-10 \mathrm{keV}$ luminosity function over the $0.01<z<3.0$ range favors the luminosity-dependent density evolution model; (d) A large cluster of AGN was found to correspond to a supercluster of galaxies detected at $z=0.14$ (Fotopoulou et al. 2016).

\section{2 | Next steps}

One of the most intriguing (thus exciting) points raised by our 2016 results is the mismatch between the observed cluster counts and the cosmological predictions from the CMB cosmology (Pacaud et al. 2016). A similar problem had independently been pointed out by the Planck cluster counts but for a much higher mass range and for scaling relations derived in a totally different manner. We are thus facing a dilemma: either there is something that we do not understand in the physics of cluster formation and evolution, or the cosmological model is different or more complicated than currently assumed. We shall use the complete cluster catalog to investigate this question in more depth. The enlarged statistical sample will allow us to test the impact of various hypotheses like the ratio $R_{500} / R_{\mathrm{c}}$ that was held fixed to 0.15 in our analysis and to proceed with the simultaneous modeling of cosmology, selection effects, and cluster evolution. We shall also benefit from the deep high-quality optical coverage of the XXL-N field by the Hyper-Suprime Camera on the Subaru telescope (HSC Wide Survey ${ }^{3}$ ), which will greatly improve the lensing determination of our cluster masses. A second data release at greater depth will occur in 2017 along with associated scientific articles. We foresee the final data release, including the cluster selection function, toward the end of 2018.

\subsection{1 | The X-ray background}

The XXL survey enables, for the first time, the study of the diffuse X-ray background (XRB) on large scales at a high 


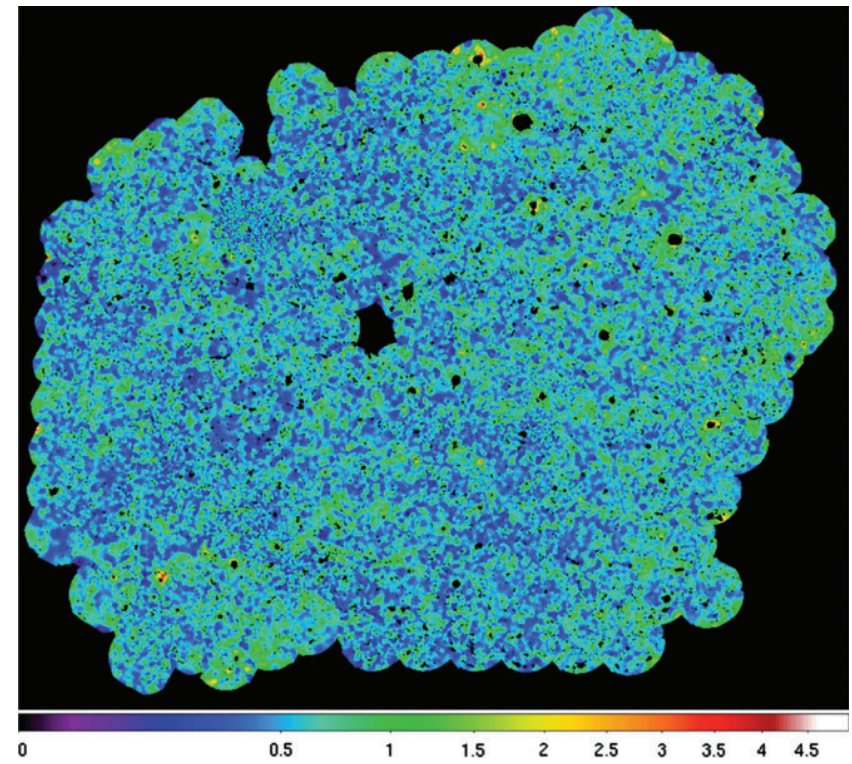

FIGURE 2 View of the X-ray background in the XXL-S field. Each circle corresponds to an XMM observation (field of view: $30^{\prime}$ ). The X-ray sources have been removed and the soft and particle backgrounds subtracted. The image is exposure-corrected and adaptively smoothed: some large-scale structure is obvious. Covered area: $25 \mathrm{deg}^{2}$; displayed band: $0.4-1.3 \mathrm{keV}$. The color scale is in unit of counts $\mathrm{s}^{-1} \mathrm{deg}^{-2}$.

angular resolution and high sensitivity (last studies were on the ROSAT All-Sky Survey data). A first impression of the scientific potential of the X-ray data is rendered in Figure 2. We are currently working on the characterization of the structures remaining after source extraction. We are undertaking an auto-correlation study of the map pixels as well as correlations between the X-ray and various maps (HI, IR, and FIR) and catalogs (optical and IR galaxies).

\subsection{2 | Numerical simulations}

When computing the cluster selection function, we assumed so far that the cluster $\mathrm{X}$-ray emission is spherically symmetric and follows a $\beta=2 / 3$ profile; the AGN population was matched to the observed $\log N-\log S$, but randomly distributed over the field (Pacaud et al. 2016). We shall switch to hydrodynamic simulations, which will provide us with more realistic cluster shapes (mergers, cool cores, etc.) and with a physical in situ modeling of the X-ray emission of the AGN population (Koulouridis et al. in preparation); an example is displayed in Figure 3. In the end, we shall compute different selection functions, depending on the AGN physics assumed and also on the cosmology. One interesting question is how much the selection function (computed in the flux vs. apparent-size plane) is dependent on the assumed cosmology.

\subsection{3 | Final cosmological analysis}

The cosmological analysis of the complete cluster sample will be performed using the traditional $d n / d M / d z$ approach. In parallel, we shall use a new method based on X-ray diagnostic diagrams of the cluster population, that is, relying on
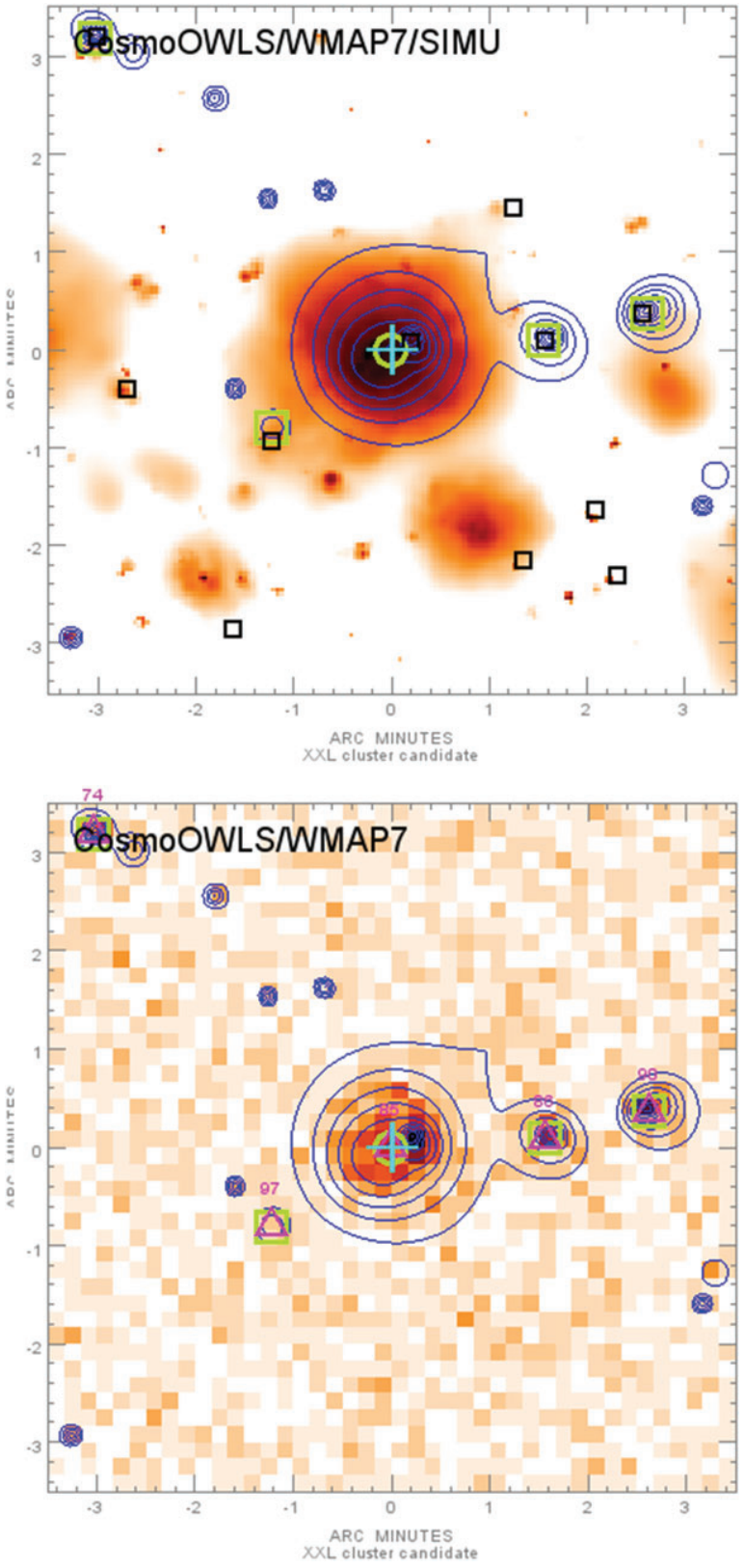

FIGURE 3 Extracted from a cosmo-OWLS lightcone, this simulated $7^{\prime} \times 7^{\prime}$ image, is centered on a $z=0.95$ cluster having a mass of $M_{500}=3.5 \times 10^{14} M_{\odot}$ Top: X-ray emissivity map in the $0.5-2 \mathrm{keV}$ band for the active galactic nuclei (AGN) 8.0 model (Le Brun et al. 2016 and references therein). The AGN X-ray luminosity is modeled following Koulouridis et al. (in preparation), and the black squares indicate AGN producing more than 15 photons. Bottom: Corresponding simulated XMM 10-ks image where all instrumental effects are taken into account: PSF, vignetting, diffuse, and particle backgrounds. The green circle indicates that the central source has been detected as extended, and the green squares stand for point-like sources.

observable quantities only: count rate, hardness ratio, apparent size, and redshift. This allows us to bypass the direct mass determination and thereby to greatly simplify the calculations. Moreover, since we deal with raw X-ray counts, we can include the entire cluster catalog in the analysis, even if those clusters are too faint to estimate their mass (Clerc et al. 2014; Pierre et al. 2016, submitted for publication). 

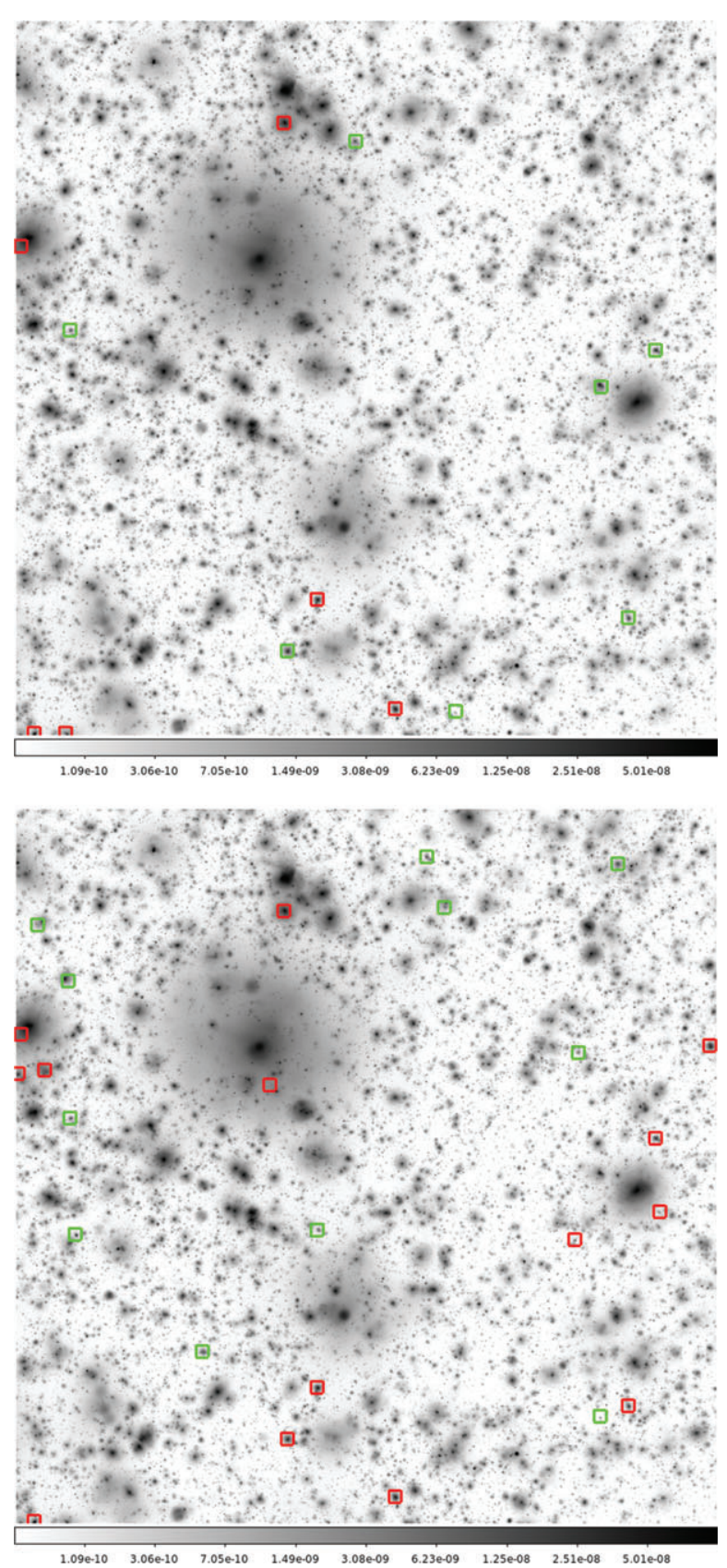

FIGURE 4 Extracted from a cosmo-OWLS active galactic nuclei (AGN) 8.0 lightcone, these simulated $3 \times 3 \mathrm{deg}^{2} \mathrm{X}$-ray emissivity images show the effect of the XMM sensitivity increase on the detectability of high-redshift clusters. The AGN X-ray emission is modeled in situ from the black-hole masses and accretion rates given by the simulation. The red and green symbols indicate the $z>1 \mathrm{C} 1$ and $\mathrm{C} 2$ detections, respectively. Top: 10-ks XXL. Bottom: 40-ks XXL-II.

\section{I PROSPECTS FOR THE NEXT DECADE}

With the new XMM operation mode using four reaction wheels, the fuel consumption is halved, which, in principle, will allow the extension of the XMM observations up to the year $\sim 2028$. An optimal use of this available time will be a matter of trade-off. While there are excellent arguments for undertaking very deep observations of well-defined samples of X-ray emitting objects, there are also compelling reasons to complete a survey of some $50 \mathrm{deg}^{2}$ at a depth of $40 \mathrm{ks}$; let us call it XXL-II. Not only will the number of detected objects be significantly higher than that achieved by XXL, but also the population of currently detected clusters and AGN will be much better characterized. This will have a very noticeable effect on the cosmological analysis (e.g., Pierre et al. 2011). In this section, we outline a few key achievements expected from such a deep uniform mosaicking. For that, we assume that the Deep HSC Survey will extend over the entire XXL-N region accordingly (discussion in progress); this will allow highly reliable and independent cluster mass measurements.

\section{1 | Characterization of the $z<0.5$ cluster population}

XXL has been very successful in the understanding of the properties of medium-high mass clusters $(T \geq 2 \mathrm{keV}$, i.e., $2 \times 10^{13}<M_{500} / M_{\odot}<10^{14}$; Pacaud et al. 2016; Giles et al. 2016; Lieu et al. 2016). The properties of the lower mass galaxy group population remain largely uncharted territory, but it is a regime in which XXL-II would have a profound impact. A key question is the degree to which groups differ from being scaled-down versions of higher mass clusters, motivated by the expectation that nongravitational processes (AGN and SN feedback) are more effective in the group-scale regime. Recent simulations have shown that scaling relations are best modeled by an evolving broken power law (Le Brun et al. 2016), highlighting the decreasing gas fraction as a function of mass (Eckert et al. 2016). However, an observational consensus of the presence of a break in the scaling relations has yet to be reached, with studies showing the group scaling relations are both consistent (Sun et al. 2009) and inconsistent (Kettula et al. 2015; Lovisari et al. 2015) with higher mass systems. The main drawback of the majority of these works is the small sample sizes and inhomogeneous samples with poorly understood selection biases. XXL-II offers the opportunity to overcome these drawbacks. At a depth of $40 \mathrm{ks}$, we would be able to measure the temperatures to $\gtrsim 30 \%$ accuracy for all groups out to $z=0.2$ above $L_{[0.5-2.0] \mathrm{keV}}=10^{42} \mathrm{erg} \mathrm{s}^{-1}$ and out to $z=0.5$ above $L_{[0.5-2.0] \mathrm{keV}}=5 \times 10^{42} \mathrm{erg} \mathrm{s}^{-1}$. This represents the crucial $T \approx 0.5-2 \mathrm{keV}$ range where feedback should dominate over gravity. The dominance of feedback in low-mass systems leads to large scatter in X-ray luminosity at fixed mass. The amount and mass dependence of this scatter are important clues to the nature of the feedback physics. Measuring the scatter can be done by studying clusters selected through non-ICM properties (e.g., optical tracers). Recent studies of optically selected clusters show an increased scatter in X-ray luminosity compared to X-ray-selected samples (Andreon et al. 2016). Indeed, many lower mass groups in the XXL-N field that are selected from the galaxy and mass assembly (GAMA) survey are undetected in current XXL data (Giles et al. in preparation). With the proposed XXL-II, we will be able to measure the full range of $L_{\mathrm{X}}$ scatter at a given mass for a complete sample of all GAMA systems with $\geq 10$ friends-of-friends members ( 75 objects). Moreover, the group 
mass range at $z \sim 0.3$ will represent the bulk of the eROSITA sample (see, e.g., Borm et al. 2014) but will be observed with an exposure time on average about an order of magnitude lower. XXL-II, with its extensive multi- $\lambda$ coverage, will provide the multiband scaling relations that eROSITA will need to fulfill its precision cosmology goal.

\subsection{Census of the $1<z<2$ clusters}

In the $z>1$ range, we are facing a situation similar to that some 20 years ago, with the Rosat All-Sky Survey (RASS) and the Palomar Observatory Sky Survey (POSS): clusters around $z=0.4$ were at the sensitivity limit and considered distant objects. Nowadays, we may replace " $z=0.4$ " by " $z=1.2$ ", "RASS" by " $10 \mathrm{ks}$ XMM" and "POSS" by "CFHTLS-Wide". The difference, though, is that we have good reasons to believe, due to comparable advances in numerical simulations, that the $1<z<2$ range corresponds to the formation epoch of massive clusters and, thus, is of extreme cosmological relevance. A few tens of X-ray clusters are known at these distances (and a couple beyond $z>2$; e.g., Gobat et al. 2011), but their space density is still undetermined because of the very heterogeneous conditions under which these detections were made. While the observed cluster evolution out to $z \sim 1$ is compatible with self-similarity, there are hints that clusters are fainter at higher redshifts. Our preliminary processing of the cosmo-OWLS AGN 8.0 simulations, duly including the X-ray AGN emission, indicates that we would detect a dozen $z>1 \mathrm{C} 1$ clusters (over $50 \mathrm{deg}^{2}$ ) with $10 \mathrm{ks}$ exposures for the WMAP7 cosmology. For the Planck 2014 cosmology, the number of high- $z$ detections is doubled; pushing to $40 \mathrm{ks}$ exposures would again double the number of detections. Finally, considering the fainter C2 population would add another factor of 2 . We should then end up with a homogeneous sample of 50-100 $z>1$ clusters, depending on the cosmology and cluster evolution rate. ${ }^{4} \mathrm{~A}$ visual impression of the sensitivity improvement is given in Figure 4. The gain expected from XXL-II is many-fold: (a) determine the density of high-redshift clusters due to the even X-ray exposure; (b) compare with that from near-infrared (NIR) observations, which tends to be much higher, and address the challenging issue of projection effects in galaxy-density-based cluster searches at high- $z$; (c) determine the properties of these objects given the extensive multi- $\lambda$ coverage (accordingly deep Chandra follow-up would be extremely useful to characterize the AGN population in distant clusters); (d) perform a stand-alone cosmological analysis based on rare-events statistics for the $1<z<2$ range. We note that the systematic exploration of this high-redshift universe at the XXL-II depth is out of the reach of the eROSITA wide survey.

${ }^{4}$ For the definition of the $\mathrm{C} 1$ and $\mathrm{C} 2$ cluster selection criteria, refer to Pacaud et al. (2016).

\subsection{Active galactic nuclei}

Under the assumption that the clustering strength of X-ray sources is independent of the survey flux limit, the increase by a factor of 2-3 of the number of sources in the $40 \mathrm{ks}$ survey (e.g., Cappelluti et al. 2009) could decrease the quasi-Poissonian uncertainties of the correlation function by a factor of at least $\sim 4$, since $\sigma_{\mathrm{w}}(\theta) \simeq \sqrt{1+w(\theta)} / D D$, with DD the number of source pairs within separations $\theta \pm \delta \theta$. However, this could be a rather optimistic reduction of the uncertainties since there is a known dependence of the X-ray source clustering amplitude to the survey flux limit (Plionis et al. 2008), with lower flux-limited samples showing weaker clustering. In addition, the number of moderate/high-redshift obscured AGN will significantly increase from a deeper exposure in XXL. Furthermore, the X-ray spectral characterization of AGN, currently limited to the brightest sources (largely dominated by unobscured/moderately obscured AGN), will largely improve, allowing for more sophisticated and physically motivated models to be adopted.

\section{4 | The X-ray background}

Increasing the exposure of the XXL survey from an average of 10 to $40 \mathrm{ks}$ will have several significant benefits for studies of the cosmic XRB in addition to the improvement in statistics (observations of the XRB are nearly always photon-limited). Longer exposures and multiple passes greatly enhance the ability to identify soft-proton flaring events and either the rejection or modeling and subtraction of their contribution from images. Additional exposure also enables the improved modeling and subtraction of the quiescent particle background. Both these improvements lead to a significant increase in the reliability of the data. They are critical due to the relative faintness of the XRB and scientific relevance of the enabled studies, for example, the search for the cosmic web. The increase in statistics will also be important, as the size of useful resolution elements will be decreased by a factor of 2, enabling the search for finer structure in the XRB.

\section{5 | CONCLUSION}

Almost two decades of XMM and Chandra observations have revolutionized much of our knowledge of clusters of galaxies. Moreover, X-ray survey analyses taught us how to handle the many issues impinging on cluster precision cosmology (selection effects, covariance between observables, mass determination, and evolutionary physics). While the publication of the final results of the 10-ks XXL survey will occur in 2 years time, we propose to start increasing its depth by a factor of 4 . Given the already existing XMM observations, the total net XMM time to reach a uniform coverage of $40 \mathrm{ks}$ over the $50 \mathrm{deg}^{2} \mathrm{XXL}$ area is of the order of $13 \mathrm{Ms}\left(45 \mathrm{deg}^{2} \times 9\right.$ pointings $\left./ \mathrm{deg}^{2} \times 30 \mathrm{ks}\right)$. This can be easily 
accommodated at a rate of 2-3 Ms over 6 years, knowing that the total available Open Time is $\sim 15 \mathrm{Ms} /$ year. The main goal is to derive competitive stand-alone cosmological constraints from the clusters and AGN present in these two particular areas. Furthermore, the global merit of the project will be greatly enhanced thanks to the synergy between the many associated surveys, from UV to radio. With the new very sensitive instruments such as the HSC in the optical and NIKA2 in the S-Z domains, the scientific potential of the dataset will serve a very large scientific community. In the same spirit, we advocate the opening of joint XMM-Chandra Very Large Programs: along with hydrodynamic simulations, this will definitively enlighten the physics and evolution of the low-mass $z \sim 0.5$ and high-redshift clusters, in relation to galactic nucleus activity. XXL-II will bridge the gap between the expected eROSITA and Athena performances in terms of combined sensitivity, coverage, and angular resolution. It will open a totally new field for XRB research and will constitute a unique legacy for the next generations, particularly for the cosmological exploitation of the eROSITA and Euclid missions.

\section{REFERENCES}

The articles from the first XXL series quoted in the present paper are indicated with roman numbers in the reference list

Allen, et al. (2011). ARA\&A, 49, 409.

Andreon, et al. (2016). $A \& A, 585,147$.

Borm, et al. (2014). $A \& A, 567,65$.

Cappelluti, et al. (2009). $A \& A, 497,635$.

Chiappetti, et al. (2013). MNRAS, 429, 1652.

Clerc, N., et al. (2014). MNRAS, 423, 356.

Eckert, D., et al. (2016). A\&A, 592, A2 (XXL paper XIII).

Elyiv, et al. (2012). $A \& A, 537,131$.

Fotopoulou, et al. (2016). A\&A, 592, A6 (XXL paper VI).

Giles, P., et al. (2016). $A \& A, 592$, A3 (XXL paper III).

Giles et al. (in preparation).

Gobat, R., et al. (2011). A\&A, 526, 133.

Kettula, K., et al. (2015). MNRAS, 451, 1460.

Koulouridis et al. (in preparation).

Le Brun A., et al. (2016). arXiv: 1606.04545.

Lieu, M., et al. (2016). A\&A, 592, A4 (XXL paper IV).

Lovisari, L., et al. (2015). $A \& A, 573,118$.

Mantz, A., et al. (2014). ApJ, 794, 157 (XXL paper V).
Pacaud, F., et al. (2016). $A \& A, 592$, A2 (XXL paper II).

Pierre, M., et al. (2011). MNRAS, 414, 1732.

Pierre, M., et al. (2016). A\&A, 592, A1 (XXL paper I).

Plionis, M., et al. (2008). ApJ, 674, L5.

Pompei, E., et al. (2016). A\&A, 592, A7 (XXL paper VII).

Sun, et al. (2009). ApJ, 693, 1142.

More information can be found on the website of: Hot Spots in the XMM Sky: Cosmology from $X$-ray to radio a prospective conference held in Mykonos (June 2016): http://www.astro.auth.gr/ xmmcosmo16

How to cite this article: Pierrel M, Adami C, Birkinshaw M, Chiappetti L, Ettori S, Evrard A, Faccioli L, Gastaldello F, Giles P, Horellou C, Iovino A, Koulouridis E, Lidman C, Le Brun A, Maughan B, Maurogordato S, McCarthy I, Miyazaki S, Pacaud F, Paltani S, Plionis M, Reiprich T, Sadibekova T, Smolcic V, Snowden S, Surdej J, Tsirou M, Vignali C, Willis J, Alis S, Altieri B, Baran N, Benoistm C, Bongiorno A, Bremer M, Butler A, Cappi A, Caretta C, Ciliegi P, Clerc N, Corasaniti PS, Coupon J, Delhaize J, Delvecchio I, Democles J, Desai S, Devriendt J, Dubois Y, Eckert D, Elyivl A, Farahi A, Ferraril C, Fotopoulou S, Forman W, Georgantopoulos I, Gugliehno V, Huynh M, Jerlin N, Jones C, Lavoie S, Le Fevre J-P, Lieu M, Kilbinger M, MaruIli F, Mantz A, McGee S, Melin J-B, Melnyk O, Moscardini L, Novak M, Piconcelli E, Poggianti B, Pomarede D, Pompei E, Ponman T, Ramos Ceja ME, Rana P, Rapetti D, Raychaudhury S, Riccil M, Rottgering H, Sahlen M, Sauvageot J-L, Schimd C, Sereno M, Smith GP, Umetsu K, Valageas P, Valotti A, Valtchanov I, Veropalumbo A, Ascaso B, Barnes D, De Petris M, Durret F, Donahue M, Ithana M, Jarvis M, Johnston-Hollitt M, Kalfountzou E, Kay S, La Franca F, Okabe N, Muzzin A, Rettura A, Ricci F, Ridl JJ, Risaliti G, Takizawa M, Thomas $\mathrm{P}$ and Truong N. The XXL survey: First results and future, Astron. Nachr./AN. 2017;338:334-341. https://doi.org/10.1002/asna.201713352. 